European Association for the Development of Renewable Energies, Environment and Power Quality (EA4EPQ)
International Conference on Renewable Energies and Power Quality (ICREPQ'11)

Las Palmas de Gran Canaria (Spain), 13th to 15th April, 2011

\title{
Investigation and Comparison between International Standards for Information Integration and Control of ECSs based on RESs over IP-based Networks
}

\author{
S. Jaloudi ${ }^{1}$, A. Schmelter ${ }^{1}$, E. Ortjohann ${ }^{1}$, W. Sinsukthavorn ${ }^{1}$, N. Alamin ${ }^{1}$, P. Wirasanti ${ }^{1}$ and D. Morton ${ }^{2}$ \\ ${ }^{1}$ Department of Electrical Engineering \\ South Westphalia University of Applied Sciencs \\ Campus of Soest - Luebecker Ring 2, D-59494 Soest (Germany) \\ Phone/Fax number:+49 2921 378439, e-mail: Ortjohann@fh-swf.de, samer_husni@yahoo.com \\ ${ }^{2}$ The University of Bolton \\ Deane Road, Bolton, BL3 5AB, U.K.
}

\begin{abstract}
Energy converting systems (ECSs) based on renewable energy sources (RESs) are penetrating rapidly in the power system including their associated software and hardware (SW/HW) systems. These systems are geographically dispersed, and related to different manufacturers. Therefore, the future power system can be considered as a distributed SW/HW system. Information integration and interoperability are two serious problems in distributed systems, which mainly include communication networks and communication protocols. The International Electrotechnical Committee (IEC) standards are proposed for this purpose and for the control as well. Indeed, some of these standard protocols can be used over IP-based WANs. However, future power systems, which contain many RESs in all voltage levels, could employ the Internet/Intranet WAN for both control and telemeter. This paper investigates the available standards related to power systems, discusses the possibility of applying some of these standard protocols for the control and telemeter of ECSs over Internet/Intranet WAN, compares between two legacy standard protocols and presents the factors for choosing the right protocol for such purposes. In addition, it covers the gaps, the last advances and projects related to these standards.
\end{abstract}

\section{Key words}

Power systems communications, Internet for renewable energy, information integration, DNP3, IEC standards.

\section{Introduction}

Energy converting systems (ECS) based on renewable energy sources (RES) are penetrating rapidly in the power system for environmental and economic reasons. Moreover, they support the conventional power plants and hence, increase the reliability of power systems. Conventional power systems include centralized power plants, the power mainly flows in one direction, and the active control takes place only in the high voltage levels. In contrast, smart power systems, as shown in Fig. 1, include centralized power plants, distributed energy resources (DERs) as well as RESs in all voltage levels, and characterized by actively controlled bidirectional power flow [1]. However, the ECS converts the energy

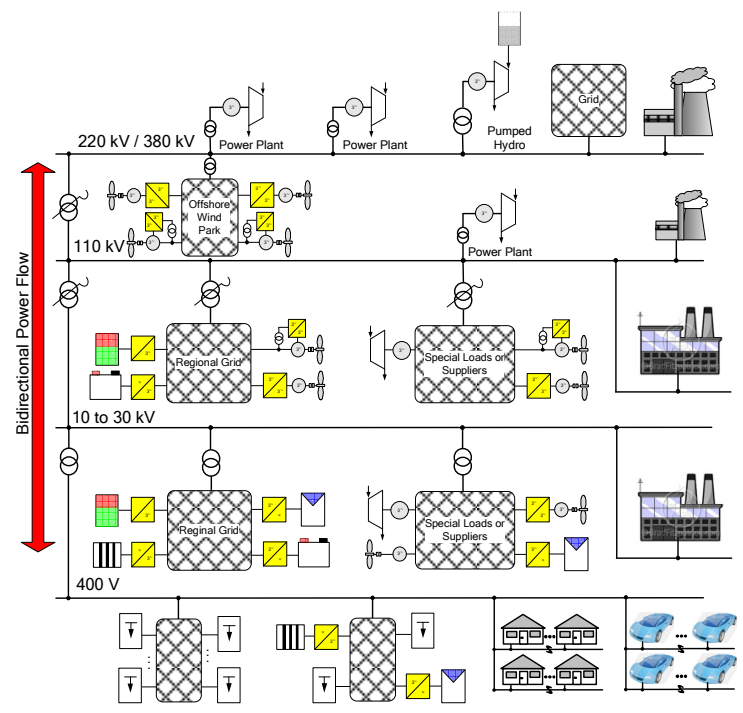

Fig. 1. A power system with actively-controlled bidirectional power flow [1].

from one form to DC current, which must be converted into AC in order to be fed to the grid. This can be done by the inverter, which is the interfacing unit that provides decoupling between frequency and voltages across the terminals of the ECS from one side and the grid voltage and frequency from the other side [2].

However, if ECSs of RESs are installed in large scale, they form power plants in the high voltage levels; otherwise they are installed in the distribution level and hence, called DERs. In both cases, some emerging international standards, mainly from the International Electrotechnical Committee (IEC), can be used for the control and telemeter over IP-based networks. While the IEC 61400-25 is proposed for data modelling in wind power plants, the IEC 62056 is proposed for telemeter and the IEC 61850 for communication and data modelling in substations, hydro power plants, and DERs.

On the other hand, the economic impact of DERs motivates many consumers to invest in this energy, and 
therefore install their own resources in the distribution level. When these resources join the grid, they form new structures in the power system that lead to changes in the system behavior. This requires that the new formed system adapt to the new circumstances and fit to the requirements of the power supply rules. As this new system contains many RESs, including their associated software and hardware (SW/HW) entities from different manufacturers that are geographically dispersed, its control and management would not become an easy task. In addition, information integration in this distributed system is a serious problem that covers communication protocols, security and interoperability of interconnected systems. In this paper, security issues will not be covered. However, the available IEC standards for RESs are introduced in section 2 . The communication networks in actual and future electric grids are discussed in section 3, and the standard protocols for conventional and future power systems are introduced in section 4 . While section 5 explains the application of communication protocols and networks for RESs, section 6 concludes the paper.

\section{Available IEC Standards for RESs}

RESs include but are not limited to wind energy, solar energy, biomass energy, hydro energy, etc. Airflows can be used to run wind turbines (WTs) that convert the kinetic energy of wind into rotational motion, and this is the reason why they are called ECSs. This rotational motion turns the rotor of a generator to produce electricity. For mass production of electricity, large scale WTs is used on shore and off shore, and therefore called wind power plants or wind farms. If these WTs are installed in the distribution level, they are DERs in the low voltage levels. However, wind power plants exist in the high voltage levels, and feed all the produced energy to the grid without active control. Recently, the IEC standard 61400-25 is proposed for data modeling of wind power plants components and for communication between these components and a supervisory control and data acquisition (SCADA) system [3].

Solar energy is collected from solar radiation via photovoltaic (PV) cells that absorb and convert it into electric current, and this is the reason why they are called ECSs. Such cells are grouped in modules and are installed in small as well as large scale. Large scale PV systems are called PV power plants, solar parks or solar farms. In addition, solar energy can be used to heat water and then collect the vapor to rotate steam generators in order to produce electricity. These are called solar thermal power plants, which exist in the high voltage levels, and feed all the produced energy to the grid without active control. Nevertheless, the IEC 61850-7420 and the IEC 61850-7-4 standards can be used together in order to build DERs logical nodes. They can be applied to small scale PV systems, if they are installed in the distribution level, and to PV central stations as well due to their modular nature [4].

Biomass is used to produce biofuels. As described in [5], biofuels exist in nature in three cases; the liquid case (such as ethanol, biodiesel and vegetable oil), the solid case (examples are wood fuel and the remaining portion of field crops) and the gas case (like animal waste and sewage). Fuel cells (FCs) can be used to convert the hydrogen of biofuels into electricity. However, the IEC standards 61850-7-4 and 61850-7-420 can be used for information modeling of FCs when installed as DERs, or central stations as well due to their modularity [4].

Water flows down of a dam is the simplest way to capture the water energy in order to convert it into electric current by rotating the rotor of many turbines to form a hydroelectric power plant. The IEC 61850-7-410 is recently proposed by the IEC for data modeling in such plants, which specifies the additional common data classes, logical nodes and data objects required for the use of IEC 61850 in a hydropower plant [6].

On the other hand, these standards can be used over IPbased networks, such as Internet/Intranet WAN, which introduce cheap infrastructure for future power systems.

\section{Communication Networks in Actual and Future Electric Grids}

The future power system can be considered as a distributed and heterogeneous system because it could contain multiple operating systems, diverse communication networks, and various ECSs based on RESs with their associated SW/HW devices from different manufacturers. Interoperability in distributed systems is a problem that mainly includes communication networks and information exchange. Communication networks used in power systems can be categorized in three main types [7]; fixed-line networks such as the public switched telephone network (PSTN), wireless networks such as the global system for mobile (GSM) communications, and computer networks such as LANs and WANs. Electric utilities employ these networks in different fields of applications; satellite communications for time synchronization through the global positioning system (GPS), WANs for real time control, monitor, and intensive data collection, etc.

Computer WANs normally follow a specific communication model, which is either open or closed. The description of the open model is available for public use, like ISO's Open System Interconnection (OSI) model, Internet model (TCP/IP protocol suite), and IEEE 802 model. The closed model is a proprietary model, like IBM's system network architecture (SNA). The users of such model are limited to the hardware and software of a specific vendor. Fig. 2 shows the open models [8-9]. The OSI model has seven layers, while the Internet model has four. The link layer of the Internet model represents the network interface to the communications infrastructure, and normally follows the IEEE 802 standard, which is the most used practical model. The IEEE model divides the datalink layer of the ISO model into two sub-layers; the logical link control (LLC) and the media access control (MAC). It deals with the hardware interfaces and LAN topologies, such as Ethernet, Bus-based, WLAN, etc. Nevertheless, real communications between layers take place only through the physical layer and in a vertical 


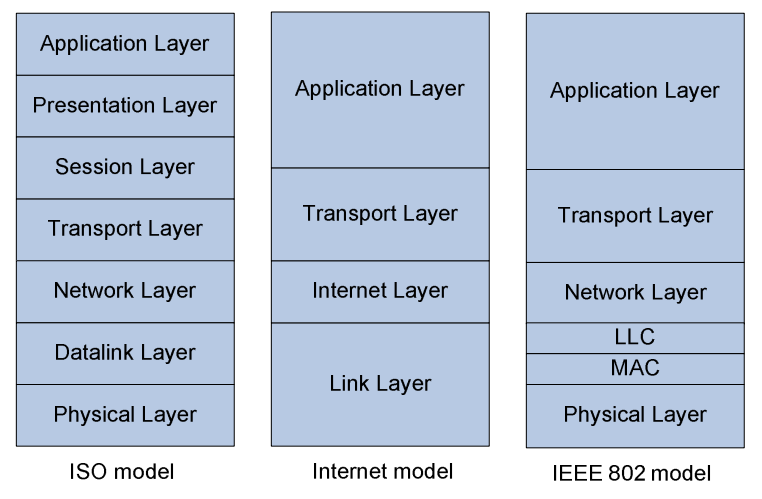

Fig. 2. Open communication models.

way, between adjacent layers. Common layers offer virtual communications through protocols and in a horizontal way. This forms a peer-to-peer communication, which makes the security a main issue when using this type of communications.

However, by using the application layer for power system communications and information integration, services, models and objects can be built without knowing the details of the network infrastructure. This allows following the state-of-the-art in the communication technology and permits the industry to concentrate their investments in the application layer. Nowadays, information exchange in many power systems use the extensible mark-up language (XML) based technologies, in the application layer, as a mediator for seamless information integration and exchange among heterogeneous systems. The XML-based messages are transferred over IP-based networks, such as the Internet. In addition, most standards of power systems, such as the standards of the IEC, are application-layer standards. This necessitates the usage of the application layer for power-related information integration.

Depending on the previous description, the IP-based communication networks will play an important role in future power systems. Computer LANs and WANs, WiMAX networks, etc. are all examples of IP-based networks. The Internet WAN is the most popular example of such networks that will offer increased bandwidth, availability, and cost-effective infrastructure among other benefits for future power systems that would include many RESs with their associated SW/HW systems. They will benefit of the Internet not only in supporting energy generation but also in reselling the excess of energy. Moreover, it will be possible to control, monitor and regulate the nodes in the power system intelligently, over the Internet (or Intranet). This type of WAN can interconnect different stakeholders, in all voltage levels of the power system, via web servers.

\section{Standard Communication Protocols for Conventional and Future Power Systems}

The application of standard communication protocols is rapidly penetrating in power systems. More and more power-related applications utilize information and communication technologies (ICTs) and standard protocols for information exchange even for mission critical purposes. The ICT intelligently integrates the SW/HW, of the new ECSs based on RESs, into the existing conventional power systems. This comes by the installation of necessary SW/HW to control, manage and monitor different devices in the grid, and by the application of standard communication protocols between different stakeholders of the power system. Some of these protocols have already been used in many power systems. The most popular are SCADA protocols, such as the IEC 60870-5-104 (T104), refer to part A, and the distributed network protocol version 3 (DNP3), refer to part B. New communication protocols are emerging such as the IEC 61400-25 for wind power plants (part C), the IEC 62056-52 for telemeter (part D), the IEC 61850 for communication and data modelling in substations, DERs and hydroelectric power plants (part E), among other standards that are recently released by the IEC.

\section{A. IEC 60870-5-104}

The IEC 60870 standard defines communication protocols between control center (CC) and substation. part 5 consists of 6 sections, under the general title "Telecontrol equipment and systems - Part 5: Transmission protocols" [10]. Part 5-104 (T104) represents a SCADA protocol and widely used for telecontrol between the $\mathrm{CC}$ and remote terminal units (RTU) in Europe. This protocol can be used over Internet WAN because it was built according to the IEEE model.

The IEC 60870-5-104 data units are application service data units (ASDUs) in the IEEE 802 model (RFC 2200), as shown in Fig 3. This means that they are services in the application layer that are divided into data units. These ASDUs must be associated with control frames, especially for information transfer. These frames are called application protocol control information (APCI). The APCI togethor with the ASDU form the application protocol data unit (APDU), which is the main transferred data unit from the CC to the RTU in the control direction, and from the RTU to the CC in the monitoring direction, as shown in Fig. 4. The minimum size of the APDU is 6 bytes, while the maximum size is 255 bytes including control and header frames [10]. This limits its usage to transfer large amount of small sized data units. However, The server (controlled station) always uses TCP port number 2404, while the client (controlling station) uses

\begin{tabular}{|c|l|}
\hline Application Layer & $\begin{array}{l}\text { IEC 60870-5-104: } \\
\text { Procedures, APCIs, ASDUs } \\
\text { (Information Objects and Elements) }\end{array}$ \\
\hline $\begin{array}{c}\text { Transport Layer: } \\
\text { TCP (RFC 793) }\end{array}$ & Port Number 2404 \\
\hline $\begin{array}{c}\text { Network Layer: } \\
\text { IP (RFC 791) }\end{array}$ & IPv4 Address \\
\hline $\begin{array}{c}\text { Datalink Layer: } \\
\text { (RFC 894) }\end{array}$ & Ethernet Driver \\
\hline $\begin{array}{c}\text { Physical Layer: } \\
\text { IEEE 802.3 }\end{array}$ & xBase-T \\
\hline
\end{tabular}

Fig. 3. IEC 60870-5-104 in the IEEE 802 model. 


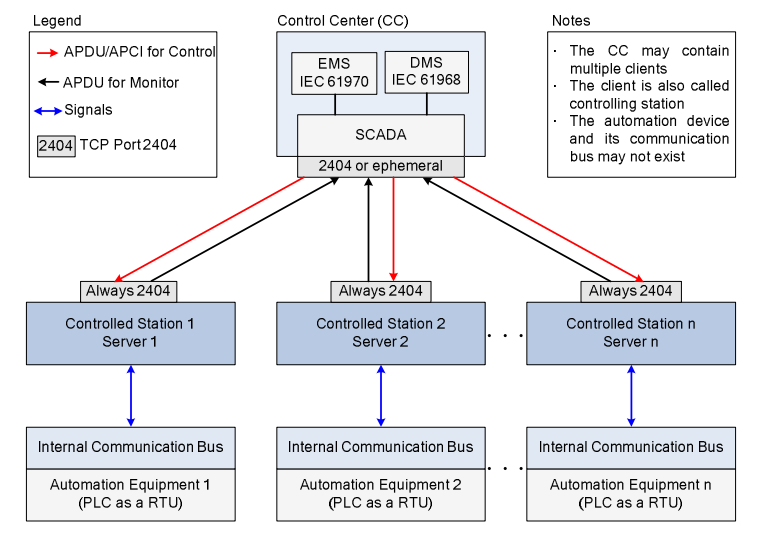

Fig. 4. The IEC 60870-5-104 protocol phylosophy.

ephemeral TCP ports. In addition, an IPv4 address must be assigned to each client/server in order to be used in a WAN such as the Internet. The hardware part of the RTU may or may not exist. This totally depends on the application that may use a database (DB) instead of a HW device, or may use both of them together in a LAN.

\section{B. DNP3}

It is the American version of the early parts of IEC 60870-5 and originally designed as a general SCADA protocol. It was developed in the 90 s while it was in public use, and later, its ownership is given to the DNP3 users group. In January 2010, the IEEE announced that work is underway for the formal recognition of DNP3 as an IEEE standard. Designated as IEEE P1815 ${ }^{\mathrm{TM}}$, the IEEE claims that the standard will promote interoperability across hundreds of operational systems with thousands of installed devices, as well as strengthening security protocols while maintaining compatibility with existing object models [11]. The IEEE proposes that it is an extremely robust and flexible methodology for optimizing data transmission between mission-critical devices in process automation settings.

\section{IEC 61850}

IEC 61850, the standard protocol for "Communication networks and systems in substations" [12], is a communication and data modeling standard for the design of substation automation systems using the object oriented data model, substation configuration language (SCL), and abstract communication service interface (ACSI) that specifies the models and services used for access to the elements of the domain specific object model. The standard divides substation communication into three levels: the substation level, which includes the human-machine interface (HMI) and interfaces with outside the substation, the bay level (unit level), which includes the protection and control intelligent electronic devices (IEDs), and the process level, which includes intelligent sensors, actuators and other I/O devices. In addition, the standard utilizes different network technologies and communication mechanisms for exchanging information in the substation, as shown in Fig 5, via several protocols:
1) Client/server communication protocol: which uses the mapping of the application models and objects to manufacturer message specification (MMS) for transmition outside the substation.

2) Generic Object Oriented Substation Events (GOOSE) protocol: for real-time messaging between substation devices

3) Sampled Analogue Values (SAV) protocol: for transmiting measured data from current and voltage transformers (CTs/VTs).

The GOOSE messages and sampled values are in use within the substation, sent from the application layer directly to the real-time Ethernet, bypassing the transport and network layers. In contrast, the client/server mechanism uses the whole stack of the ISO model, uses the specific communication service mapping (SCSM) for mapping the data models to a communication protocol, and can be synchronized via simple network time protocol (SNTP). All these properties make it suitable for SCADA systems. However, the MMS, by itself, is not the right solution as a communication protocol for SCADA systems and hence, the mapping to a legacy SCADA protocol, such as the DNP3 and T104 is proposed over WANs, as will be explained in section 5 .

\section{IEC $61400-25$}

According to [3], the IEC 61400-25 series defines communications for monitoring and control of wind power plants. The architecture of this standard series has been selected to provide an abstract definition of classes and services such that the specifications are independent of specific protocol stacks, implementations, and operating systems. It mainly includes:

1) Part 25-1: description of principles and models

2) Part 25-2: information models

3) Part 25-3: information exchange models

4) Part 25-4: mapping to communication profile

5) Part 25-5: conformance Testing

6) Part 25-6: logical node classes and data classes for condition monitoring

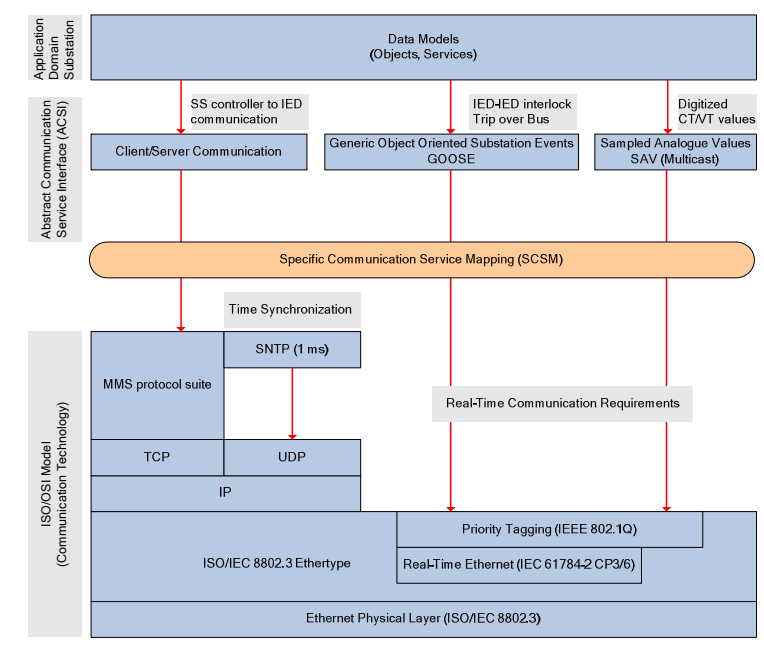

Fig. 5. Approach of the IEC 61850 protocol stack. 
Part 25-4 specifies the mappings to protocol stacks of MMS, DNP3, T104, web services and OPC XML-DA. Hence, the focus of the IEC 61400-25 series is on the communications between wind power plant components such as WTs and actors such as SCADA systems. Internal communication within wind power plant components is outside the scope of this standard [3].

\section{E. IEC 62056}

The IEC 62056 is a standard for "Electricity metering data exchange for meter reading, tariff and load control". Part 52, communication protocols for management distribution line message specification (DLMS) server, is dedicated for electricity supply systems including electric energy meters in buildings, emergency electrical supplies, etc. [13]. This part is used for load telecontrol and telemeter in a SCADA, which makes it compatible with telemeter between the DER meterng equipment and the control center. Part 47 of the standard specifies the communication over IPv4 networks such as the Internet.

\section{Communication Networks and Protocols for RESs and DERs}

As mentioned above, information integration in distributed power systems mainly covers communication networks and standard communication protocols. Therefore, the available standard protocols that can be applied for communication between the control center and SW/HW systems (RTU/IED, Meter, DB) of an ECS, over an IP-based WAN, are shown in Fig. 6. While the IEC 61850-7-4 and 61850-7-420 standards define information models for the communication with DERs logical nodes, the IEC $61850-7-410$ is used for the same purpose in hydroelectric power plants. In addition, the IEC 61400 parts 25-2 and 25-3 are used for information modeling and information exchange modeling respectively in wind power plants. For the communication from the power plant or DERs to a control center, a legacy SCADA protocol, such as T104 or DNP3, is proposed. Therefore, mapping between the IEC 61850 data models and the legacy SCADA protocol is a must. For the wind power plant, this is specified by the IEC 61400-25-4 standard. For IEC 61850-7-4/420, the standard IEC 61850-80-1 can be used for the mapping to T104, and for mapping to DNP3, the National Institute of Standards and Technology (NIST) has recently proposed a project for this purpose [14]. In addition, the IEC 62056 standard can be applied to build a DER meter equipment and part 47 of the same standard can be used for the communication with the control center. However, each DER is shown in a LAN together with its related SW/HW systems as mentioned in section 4, part A.

SCADA/EMS (Energy Management System) supervises, controls, optimizes and manages generation and transmission systems. SCADA/DMS (Distribution Management System) performs the same functions for power distribution networks. Both systems enable utilities to collect, store and analyze data from hundreds of thousands of data points in national or regional networks, perform network modeling, simulate power operation, pinpoint faults, preempt outages, and participate in energy trading markets [15]. In general, the main functionalities of the control center can be summarized as follows: load frequency control (LFC), automatic generation control (AGC), economic

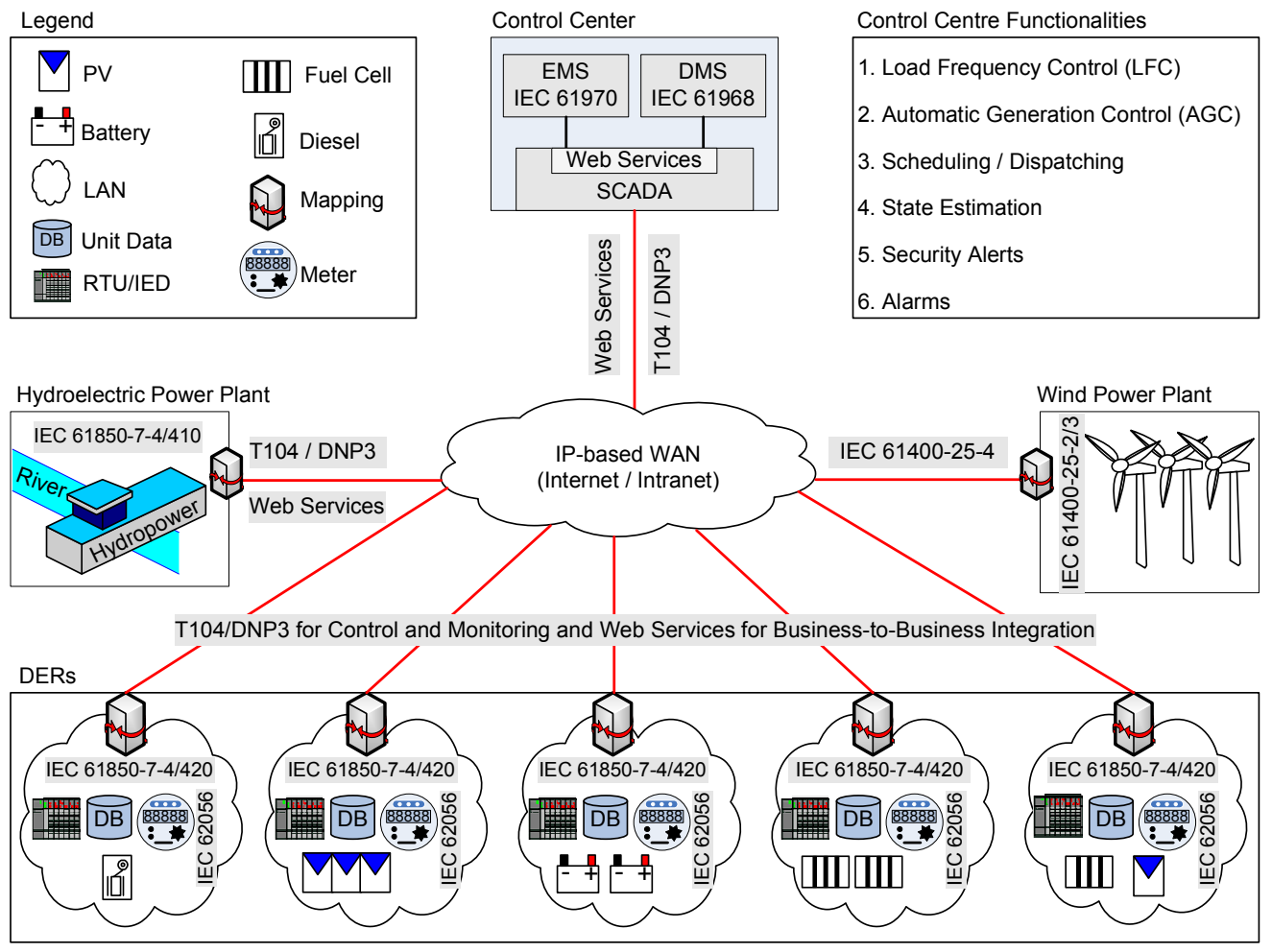

Fig. 6. Standards that can be used for the control of ECSs based on RESs and DERs over IP-based Networks. 
dispatching, state estimation, contingency analysis, security alerts, and alarms [16]. All these control functionalities can be achieved via the standards shown in Fig. 6, by mapping the data models of IEC 61850 and 61400-25 to a legacy SCADA protocol. In order to achieve business to business information integration, mapping to web services is proposed as well.

Choosing the right legacy protocol, for mapping, depends on many factors. Although both have the same origin, which is IEC 60870-5-101, the DNP3 is a general SCADA protocol. On the other hand, the amount of data to be sent and received from the DER or power plant, to the SCADA system may influence the efficiency of the whole system. While, the maximum size of the APDU in the T104 is 255 bytes including the header and control bytes, its minimum size is 6 bytes. In the DNP3, the size of the APDU may vary between 0 and 2048 bytes. Hence, T104 may require a large number of ASDUs, which may lead to a loss of efficiency, in this case.

Another important difference between both protocols is the type of data sent in each ASDU. While DNP3 allows multiple data objects in one ASDU frame, only data objects of the same type can be transmitted in one T104 ASDU frame. In contrast to T104 that uses only TCP connections, DNP3 can use both TCP and UDP connections. In addition, the IEEE has recently adopted the DNP3, as mentioned in section 4, under the name IEEE P1815 ${ }^{\mathrm{TM}}$. On the other hand, DNP3 and T104 have both certification and testing that ensure interoperability between products of different manufacturers.

Which protocol to be chosen, depends on the location (America or Europe) of the application and on the needs of the customer, especially the amount of traffic between the control center and the ECS. However, support of both protocols, for a product, would be an advantage.

\section{Conclusion}

The rapid penetration of ECSs based on RESs introduces challenges in the control and interoperability of their associated SW/HW devices and entities. By using the application layer, of the IP-based WAN, for power system communications and information integration, services, models and objects can be built, without knowing the details of the network infrastructure. This allows following the state-of-the-art in the communication technology. In addition, most international standards of power systems, such as the IEC standards, are application-layer standards. In this paper, the data models and services of the IEC 61850 standard are chosen for data modeling the logical nodes of ECSs based on RESs. As a communication protocol, legacy SCADA protocols, such as DNP3 and T104, are chosen for this purpose. Which legacy protocol to be chosen, depends on the application, especially the amount of traffic between the control center and the RES controller. Yet, another problem appears that is the mapping from data models of IEC 61850 to the legacy SCADA protocol. Some of these have already been standardized, others are under work, and some are not standardized yet.

\section{Acknowledgement}

This research work is supported by the German "Federal Ministry of Education and Research" and administrated by "Arbeitsgemeinschaft industrieller Forschungsvereinigungen "Otto von Guericke" e.V. (AiF)".

\section{References}

[1] E. Ortjohann, W. Sinsukthavorn, A. Mohd, M. Lingemann, N. Hamsic, and D. Morton, "A Hierarchy Control Strategy of Distributed Generation Systems", IEEE Int. Conf. on Clean Electrical Power, Capri, 2009, pp. 310-315.

[2] A. Mohd, E. Ortjohann, W. Sinsukthavorn, M. Lingemann, N. Hamsic, D. Morton, "Inverter-based Distributed Generation Control using Droop/Isochronous Load Sharing," PP and PS IFAC Symposium on Power Plants and Power Systems Control, Finland, July 2009.

[3] IEC 61400-25: Communications for monitoring and control of wind power plants.

[4] IEC 61850-7-420: Basic communication structureDistributed energy resources logical nodes.

[5] W. El-Kattan, M.M.A. Salama, "Distributed generation technologies: definitions and benefits", Electric Power Systems Research, Elsevier, 71, 2004, pp. 119-128.

[6] IEC 61850-7-410: Hydroelectric power plants Communication for monitoring and control.

[7] Mohammad Shahidehpour and Yaoyu Wang, Communications and control in power systems, IEEE Press Series on Power Engineering, (2003), pp. 6-21.

[8] The Internet Engineering Task Force (IETF). (1989) RFC 1122, Requirements for Internet Hosts -- Communication Layers. A website online available at http://www.ietf.org/rfc/rfc1122.txt?number=1122.

[9] L. M. Thompson, Industrial Data Communications, ISA, 4th ed., (2008), pp. 27-41.

[10] IEC 60870-5-104: Transmission protocols - Network access for IEC 60870-5-101 using standard transport profiles.

[11] An IEEE announcement "IEEE lays groundwork for adoption of DNP3 protocol as new IEEE standard". A web site online available at http://standards.ieee.org/announcements/2010/pr_DNP3newstandard.html. Last accessed Sep. 2010.

[12] IEC 61850-SER: Communication networks and systems in substations - ALL PARTS.

[13] IEC 62056-52: Electricity metering -Data exchange for meter reading, tariff and load control - Part 52: Communication protocols management distribution line message specification (DLMS) server.

[14] IEC 61850 Objects/DNP3 mapping (6.2.2). A proposed project of NIST in 2009. A web site online available at http://www.nist.gov/smartgrid/upload/12-IEC_61850_DNP3_Mapping.pdf. [15] The evolution of SCADA/EMS (from ABB). A web site available online at http://www.abb.com/cawp/gad02181/7ff771bfb4d81e96c1256e390052582f.aspx. Last accessed Sep. 2010.

[16] Anjan Bose, "Power System Control Centers: New Vision”, EPCC workshop, Duplin, Ireland, June 2009. 